\title{
Asociación de depresión y resultados funcionales en pacientes tratados quirúrgicamente por mielopatía espondilótica cervical mediante abordaje anterior
}

\author{
Association of depression and functional outcomes in patients treated surgically for \\ cervical spondylotic myelopathy through an anterior approach
}

\author{
Guadalupe F. Alcocer', Alfredo J. Moheno-Gallardo', Eulalio Elizalde-Martínez', David Rojano-Mejía², \\ Estela López-Martínez ${ }^{1}$, Karen G. González-Andrade ${ }^{1}$ y Jorge Quiroz-Williams ${ }^{3 *}$ \\ ${ }^{1}$ Departamento de Columna, Hospital de Ortopedia; ${ }^{2}$ División de Investigación en Salud; ${ }^{3}$ Coordinación Clínica de Educación e Investigación en \\ Salud, Hospital de Ortopedia. Unidad Médica de Alta Especialidad Traumatología, Ortopedia y Rehabilitación Dr. Victorio de la Fuente Narváez, \\ Instituto Mexicano del Seguro Social. Ciudad de México, México
}

\section{Resumen}

Objetivo: Determinar la asociación de depresión con los resultados clínicos en pacientes tratados quirúrgicamente por mielopatía espondilótica cervical (MEC) mediante abordaje anterior. Método: Se realizó un estudio observacional en pacientes con MEC. Se utilizaron la escala de Beck, la escala modificada de la Japanese Orthopaedic Association (mJOA), el índice de discapacidad del cuello (NDI) y la escala visual análoga (EVA) de forma prequirúrgica, al mes y 3 meses posterior a la cirugía. Resultados: La EVA inicial mostró mayor gravedad en los pacientes con depresión. Al mes y al tercer mes posteriores a la cirugía hay una disminución significativa del dolor en el grupo sin depresión $(p=0.03)$. En la mJOA al mes y a los 3 meses se observó que la gravedad disminuyó en ambos grupos, siendo más notorio en el grupo sin depresión $(p=0.02)$. El NDI prequirúrgico fue mayor en el grupo con depresión. A los 3 meses, en ambos grupos la mejoría fue notoria con respecto al grado de incapacidad preoperatorio. Conclusiones: Existe una relación favorable en los pacientes con MEC sometidos a tratamiento quirúrgico entre la ausencia de depresión previa a cirugía y la evolución clínica, con la posibilidad de promover un manejo multidisciplinario previo a la cirugía en los pacientes con depresión.

Palabras clave: Columna. Vértebra cervical. Depresión. Mielopatía cervical.

\begin{abstract}
Objective: To determine the association of Depression with clinical outcomes in patients treated surgically for cervical spondylotic myelopathy (CSM) using an anterior approach. Method: An observational study was conducted in patients with ECM. The Beck scale, modified scale of the Japanese Orthopedic Association (mJOA), neck disability index (NDI) and the Visual Analogue Scale (VAS) were used preoperatively, one month and 3 months after surgery. Results: Initial VAS showed more severe degrees in patients with depression. At one month and third month after surgery, there was a significant decrease in pain in the group without depression $(p=0.03)$. The $m J O A$ at one month and three months was observed that the degree of
\end{abstract}

\footnotetext{
Correspondencia:

*Jorge Quiroz-Williams

Avda. Diagonal Defensores de la República, SN

Col. Amor C.P. 72140 ,

Fecha de recepción: 18-08-2020

Puebla, Pue., México

E-mail: jquiwill@ hotmail.com

Cir Cir. 2021;89(5):657-663

Contents available at PubMed www.cirugiaycirujanos.com 0009-7411/@ 2020 Academia Mexicana de Cirugía. Publicado por Permanyer. Este es un artículo open access bajo la licencia CC BY-NC-ND (http://creativecommons.org/licenses/by-nc-nd/4.0/).
} 
severity decreased in both groups, being more noticeable in the group without depression $(p=0.02)$. Presurgical NDI was higher in the group with depression. At three months in both groups the improvement was noticeable with respect to the degree of preoperative disability. Conclusions: There is a favorable relationship in patients with ECM undergoing surgical treatment in the absence of depression prior to surgery and clinical evolution, with the possibility of promoting multidisciplinary management prior to surgery in patients with depression.

Key words: Spine. Cervical vertebrae. Depression. Cervical myelopathy.

\section{Introducción}

La mielopatía espondilótica cervical (MEC) es una condición degenerativa de la médula espinal que ocasiona deterioro neurológico secundario al estrechamiento del canal espinal debido a la degeneración de estructuras de la columna cervical ${ }^{1,2}$. Es la principal causa de disfunción medular en la población adulta en el mundo ${ }^{2,3}$. Causa deterioro neurológico y declive funcional que conducen a una menor independencia y peor calidad de vida ${ }^{4}$. Su incidencia exacta y su prevalencia son desconocidas. Afecta más a hombres que a mujeres, en una proporción 2-7:11,4,5. La edad promedio del diagnóstico es de 64 años $^{1,5}$. Los niveles más comúnmente afectados son $\mathrm{C} 5$ y $\mathrm{C} 6^{1,5}$. Su progresión tiende a ser lenta, con una disminución gradual o un largo periodo de inactividad. La incapacidad se establece pronto en el curso de la enfermedad y después se sigue de muchos años con periodos estáticos. Los cambios sensitivos y esfinterianos son transitorios, mientras que los cambios motores tienden a persistir y progresar con el tiempo². Los pacientes tratados con manejo conservador empeoran con respecto a su capacidad para llevar a cabo las actividades de la vida cotidiana; por el contrario, los pacientes intervenidos mejoran sus síntomas neurológicos, dolor y estado funcional ${ }^{1,2}$.

Existen numerosas escalas para la valoración del deterioro neurológico, pero las más utilizadas son la escala modificada de la Japanese Orthopedic Association $(\mathrm{mJOA})^{6}$ y la escala de Nurick ${ }^{7}$. El índice de discapacidad del cuello (NDI) es una herramienta de evaluación de la calidad de vida, específica para enfermedades de la columna cervical ${ }^{8}$.

El tratamiento quirúrgico es el método de elección en casos de mielopatía clínicamente evidente, pero existen evidencias a favor del tratamiento quirúrgico precoz en pacientes con signos leves de mielopatía. El objetivo es una adecuada descompresión de la médula espinal ${ }^{9,10}$.

Hay diferentes respuestas emocionales y conductuales a la enfermedad. El dolor de espalda se asocia también con trastornos psicogénicos, incluida la enfermedad depresiva ${ }^{11}$. La reincidencia del dolor después de la cirugía de disco puede deberse a varios factores demográficos, sociales y psicológicos, incluyendo trastornos depresivos, ansiedad, estado mental y personalidad del paciente ${ }^{12,13}$.

La MEC, como condición degenerativa, se asocia a trastornos emocionales como la depresión reactiva, que puede ser una respuesta tanto al dolor crónico y a las subsiguientes limitaciones que resultan del dolor y la limitación del movimiento y la función, como a la pérdida de empleo, vida social y amigos. Se ha reportado una correlación significativa entre la intensidad del dolor de cuello y la depresión, pero existen pocos estudios sobre los factores psicosociales y el resultado de la cirugía para la enfermedad degenerativa del disco cervical ${ }^{14}$.

Actualmente se desconoce si el tratamiento de la depresión antes de la cirugía con cualquier psicoterapia o la medicación antidepresiva pueden mejorar los resultados después de la cirugía cervical. Sin embargo, en los estudios en que se realizó terapia cognitivo-conductual en pacientes con lumbalgia crónica presentan una disminución muy significativa en la intensidad del dolor y de la discapacidad generada por la lumbalgia ${ }^{15}$.

El interés por realizar este estudio se basa en la importancia de la evaluación psicológica de los pacientes que están siendo considerados para cirugía por un proceso degenerativo de la columna cervical, ya que al ser diagnosticados previamente y recibir tratamiento psicológico mejorarán la percepción del dolor, la funcionalidad y el grado de incapacidad. El objetivo de este estudio es determinar la asociación de los síntomas depresivos con los resultados clínicos y funcionales en pacientes tratados quirúrgicamente por MEC mediante abordaje anterior.

\section{Método}

Estudio observacional descriptivo en pacientes con MEC a quienes se realiza un abordaje quirúrgico 
Tabla 1. Caracterización demográfica de los pacientes con mielopatía espondilótica cervical pertenecientes al departamento clínico y el departamento de columna del Hospital de Ortopedia de la Unidad Médica de Alta Especialidad de Traumatología, Ortopedia y Rehabilitación Dr. Victorio de la Fuente Narváez

\begin{tabular}{|c|c|c|c|}
\hline Variables & $\begin{array}{c}\text { Con síntomas depresivos } \\
(50 \%)\end{array}$ & $\begin{array}{c}\text { Sin síntomas depresivos } \\
(50 \%)\end{array}$ & $p$ \\
\hline Edad en años, media (DE) & $58.9(14.1)$ & $59.6(14.1)$ & $0.20^{*}$ \\
\hline $\begin{array}{l}\text { Sexo } \\
\text { Hombre, n (\%) } \\
\text { Mujer, n (\%) }\end{array}$ & $\begin{array}{l}11(57.9) \\
8(42.1)\end{array}$ & $\begin{array}{c}11(57.9) \\
8(42.1)\end{array}$ & $0.63^{+}$ \\
\hline $\begin{array}{l}\text { Escolaridad } \\
\text { Ninguna, n (\%) } \\
\text { Primaria, n (\%) } \\
\text { Secundaria, n (\%) } \\
\text { Media superior, n (\%) } \\
\text { Licenciatura, n (\%) }\end{array}$ & $\begin{array}{c}1(5.3) \\
5(26.3) \\
7(36.8) \\
6(31.6) \\
-\end{array}$ & $\begin{array}{l}2(10.5) \\
5(26.3) \\
4(21.1) \\
5(26.3) \\
3(15.8)\end{array}$ & $0.25^{+}$ \\
\hline $\begin{array}{l}\text { Situación laboral } \\
\text { Ama de casa, n (\%) } \\
\text { Activo, n (\%) } \\
\text { Desempleado, n (\%) } \\
\text { Jubilado, n (\%) }\end{array}$ & $\begin{array}{l}9(47.4) \\
4(21.1) \\
2(10.5) \\
4(21.1)\end{array}$ & $\begin{array}{c}10(52.6) \\
4(21.1) \\
1(5.3) \\
4(21.1)\end{array}$ & $0.94^{+}$ \\
\hline
\end{tabular}

anterior. El protocolo se presentó ante los comités de investigación y ética en investigación, con número de registro R-2019-3401-016. El estudio se realizó del 1 al 31 de mayo de 2019.

Los pacientes se captaron en la consulta externa, se les dio una plática informativa, firmaron el consentimiento informado, se les aplicaron los cuestionarios de depresión y ansiedad de Beck, las escalas de Nurick y mJOA, y el NDI, y además se evaluó el dolor con la escala visual análoga (EVA) de forma prequirúrgica, al mes y a los 3 meses de la cirugía. Los datos se analizaron con la prueba $t$ de Student.

\section{Resultados}

Se reclutaron 38 pacientes con diagnóstico de MEC que cumplían los criterios de inclusión, pero cuatro se perdieron en el seguimiento, con lo que quedó una muestra de 34 pacientes.

En la caracterización demográfica de nuestra población, en cuanto a si los pacientes presentaban o no síntomas de depresión previa a la cirugía evaluada con el cuestionario de Beck (Tabla 1), observamos una media de edad similar para ambos grupos (59 años) y un predominio del sexo masculino. Asimismo, no hallamos diferencias estadísticamente significativas en la distribución por sexo, escolaridad y ocupación.
En general, la mayoría de los pacientes no presentaban ninguna comorbilidad. Sin embargo, las enfermedades que presentaban los pacientes se distribuyeron de la siguiente forma: en el grupo sin síntomas depresivos prevalecieron los pacientes con hipertensión arterial y aquellos que además de ser hipertensos eran diabéticos (15.8\%); y en el grupo sin síntomas depresivos, la mayoría no presentaban ningún tipo de comorbilidad (68.4\%) y el resto tenían hipertensión arterial sistémica (26.3\%) y diabetes mellitus (26.3\%). Según los niveles de afectación cervical, se encontraron 10 pacientes $(26.3 \%)$ con afectación a nivel C4-C5, 5 (13.1\%) con afectación a nivel de C5-C6 y C5-C7, 4 (10.1\%) con afectación de los niveles C3-C4 y C3-C6, 3 (7.8\%) con afectación de C4-C6 y C4-C7, y 1 (2.6\%) con afectación de C2-C3, C3-C5, C3-C7 y C6-C7. En la mayoría de los pacientes se intervinieron más de dos niveles cervicales (Tabla 2).

Respecto al estado basal del grado de dolor evaluado con la EVA, en los pacientes sin síntomas depresivos se observó que la mayoría $(n=15)$ presentaban dolor de leve a moderado (Tabla 3), mientras que en los pacientes con síntomas depresivos la mayoría tenían un dolor de moderado a grave $(n=14)$. En la evaluación al mes y al tercer mes posteriores a la cirugía, solo cuatro y tres de los pacientes, respectivamente, manifestaron tener un dolor 
Tabla 2. Niveles de intervención quirúrgica en los pacientes con mielopatía espondilótica cervical pertenecientes al departamento de columna del Hospital de Ortopedia de la Unidad Médica de Alta

\begin{tabular}{lccc}
\hline Número de niveles intervenidos & $\mathbf{n}$ & $\%$ & $\mathbf{p}$ \\
\hline Sin síntomas depresivos & & & \\
1 & 5 & 27.8 & $0.035^{\star}$ \\
2 & 6 & 33.3 & \\
3 & 5 & 27.8 & \\
4 & 2 & 11.1 & \\
Total & 18 & 100 & \\
Con síntomas depresivos & & & \\
1 & & & \\
2 & 4 & 22.2 & \\
3 & 10 & 55.6 & \\
Total & 4 & 22.2 & \\
\hline
\end{tabular}

Especialidad de Traumatología, Ortopedia y Rehabilitación Dr. Victorio de la Fuente Narváez

Test de Friedman.

Tabla 3. Asociación entre los síntomas depresivos (Beck) previos a la cirugía y el grado de afectación prequirúrgico y posquirúrgico (al mes y a los 3 meses) medido mediante EVA en pacientes con mielopatía espondilótica cervical intervenidos mediante abordaje anterior

\begin{tabular}{|c|c|c|}
\hline & $\begin{array}{c}\text { Sin síntomas } \\
\text { depresivos } \\
(n=19)(50 \%)\end{array}$ & $\begin{array}{c}\text { Con síntomas } \\
\text { depresivos } \\
(n=19)(50 \%)\end{array}$ \\
\hline & n (\%) & n (\%) \\
\hline \multicolumn{3}{|c|}{$\begin{array}{l}\text { EVA prequirúrgica } \\
\text { Grado de dolor }\end{array}$} \\
\hline Leve & $7(36.8)$ & $5(26.3)$ \\
\hline Moderado & $8(42.1)$ & $9(47.4)$ \\
\hline Grave & $4(21.1)$ & $5(26.3)$ \\
\hline Total & $19(100)$ & $19(100)$ \\
\hline \multicolumn{3}{|c|}{$\begin{array}{l}\text { EVA } 1 \text { mes poscirugía } \\
\text { Grado de dolor }\end{array}$} \\
\hline Leve & $9(52.0)$ & $8(47.1)$ \\
\hline Moderado & $5(29.4)$ & $8(47.1)$ \\
\hline Grave & $3(17.6)$ & $1(5.9)$ \\
\hline Total & $17(100)$ & $17(100)$ \\
\hline \multicolumn{3}{|c|}{$\begin{array}{l}\text { EVA } 3 \text { meses poscirugía } \\
\text { Grado de dolor }\end{array}$} \\
\hline Leve & $11(64.7)$ & $8(47.1)$ \\
\hline Moderado & $6(35.3)$ & $6(35.3)$ \\
\hline Grave & ------ & $3(17.6)$ \\
\hline Total & $17(100)$ & $17(100)$ \\
\hline$p$ & $0.03^{\star \dagger}$ & $0.17^{\star \dagger}$ \\
\hline
\end{tabular}

grave en comparación con los nueve pacientes que inicialmente (EVA preoperatoria) habían manifestado un dolor con esa intensidad. La mejoría más evidente en el grado de dolor se notó en el grupo sin síntomas depresivos $(p=0.03)$.
Tabla 4. Asociación entre los síntomas depresivos (Beck) previos a la cirugía y el grado de afectación prequirúrgico y posquirúrgico (al mes y a los 3 meses) medido con la mJOA en pacientes con mielopatía espondilótica cervical intervenidos mediante abordaje anterior

\begin{tabular}{|c|c|c|}
\hline & $\begin{array}{c}\text { Sin síntomas } \\
\text { depresivos } \\
(n=19)(50 \%)\end{array}$ & $\begin{array}{c}\text { Con síntomas } \\
\text { depresivos } \\
(n=19)(50 \%)\end{array}$ \\
\hline & n (\%) & n (\%) \\
\hline \multicolumn{3}{|c|}{ mJOA prequirúrgica } \\
\hline \multicolumn{3}{|c|}{ Grado de afectación } \\
\hline Leve & $6(32)$ & $2(11)$ \\
\hline Moderado & $7(36)$ & $8(42)$ \\
\hline Grave & $6(32)$ & $9(47)$ \\
\hline Total & $19(100)$ & $19(100)$ \\
\hline \multicolumn{3}{|c|}{ mJOA 1 mes poscirugía } \\
\hline \multicolumn{3}{|c|}{ Grado de afectación } \\
\hline Leve & $6(35)$ & $3(18)$ \\
\hline Moderado & $6(35)$ & $6(35)$ \\
\hline Grave & $5(29)$ & $8(47)$ \\
\hline Total & $17(100)$ & $17(100)$ \\
\hline \multicolumn{3}{|c|}{$\begin{array}{c}\text { mJOA } 3 \text { meses poscirugía } \\
\text { Grado de afectación }\end{array}$} \\
\hline Leve & $8(47)$ & $4(23.5)$ \\
\hline Moderado & $7(41)$ & $9(53)$ \\
\hline Grave & $2(12)$ & $4(23.5)$ \\
\hline Total & $17(100)$ & $17(100)$ \\
\hline$p$ & $0.02^{\star+}$ & $0.176^{*}$ \\
\hline
\end{tabular}

En cuanto a la evaluación preoperatoria del grado de afectación evaluado con la escala mJOA, en los pacientes sin síntomas depresivos se observó que seis presentaban una afectación grave, mientras que nueve pacientes del grupo con síntomas depresivos mostraron este grado de afectación (Tabla 4). No obstante, en la evaluación al mes y a los 3 meses de la intervención quirúrgica se observó que la gravedad disminuyó en ambos grupos, siendo la diferencia más notable en el grupo sin síntomas depresivos ( $p=0.02)$.

El grado de incapacidad prequirúrgica fue de mayor gravedad en el grupo con síntomas depresivos $(n=9 ; 47 \%)$ que en el grupo sin síntomas depresivos $(n=1 ; 5 \%$ ). Por otro lado, al evaluar el grado de incapacidad al mes se observó que aumentó el número de pacientes con incapacidad grave entre los pacientes sin síntomas depresivos $(n=4 ; 24 \%)$ y disminuyó el grado de incapacidad en el grupo con síntomas depresivos ( $n=3 ; 17 \%$ ). Sin embargo, a los 3 meses, en ambos grupos la mejoría fue notoria con respecto al grado de incapacidad preoperatoria (Tabla 5). 
Tabla 5. Asociación entre los síntomas depresivos (Beck) previos a la cirugía y el grado de afectación prequirúrgico y posquirúrgico (al mes y a los 3 meses) medido mediante NDI en pacientes con mielopatía espondilótica cervical intervenidos mediante abordaje anterior

\begin{tabular}{ccc}
\hline & $\begin{array}{c}\text { Sin síntomas } \\
\text { depresivos } \\
(n=19)(50 \%)\end{array}$ & $\begin{array}{c}\text { Con síntomas } \\
\text { depresivos } \\
(n=19)(50 \%)\end{array}$ \\
\hline
\end{tabular}

NDI prequirúrgico

Grado de incapacidad

Sin incapacidad

Leve

Moderada

Grave

Completa

Total

NDI 1 mes poscirugía

Grado de incapacidad

Sin incapacidad

Leve

Moderada

Grave

Completa

Total

NDI 3 meses poscirugía

Grado de incapacidad

Sin incapacidad

Leve

Moderada

Grave

Completa

Total

p

${ }^{*}$ Test de Friedman

tEstadísticamente significativo por $p<0.05$

Algo interesante que fue posible observar en el presente estudio es que la frecuencia de depresión incrementó en los grupos con y sin síntomas depresivos en la evaluación preoperatoria (Tabla 6).

\section{Discusión}

El presente estudio contó con un perfil de población demográficamente homogéneo, incluyendo población con medias sin diferencias significativas, así como una distribución homogénea para el nivel de escolaridad y el estado laboral actual, lo que permitió realizar una estadística sin sesgo en las características demográficas.

González Caro, et al. ${ }^{15}$ y Groves y Muskin ${ }^{16}$ mencionan que las intervenciones psicoeducativas son especialmente útiles antes de intervenciones médicas
Tabla 6. Evaluación de los síntomas depresivos (Beck) al mes y a los 3 meses de la intervención quirúrgica de los pacientes con mielopatía espondilótica cervical

\begin{tabular}{|c|c|c|}
\hline & $\begin{array}{l}\text { Sin síntomas } \\
\text { depresivos } \\
(n=19)(50 \%)\end{array}$ & $\begin{array}{c}\text { Con síntomas } \\
\text { depresivos } \\
(n=19)(50 \%)\end{array}$ \\
\hline & $\mathrm{n}(\%)$ & n (\%) \\
\hline \multicolumn{3}{|c|}{ Depresión 1 mes poscirugía } \\
\hline \multicolumn{3}{|c|}{ Grado de depresión } \\
\hline Sin depresión & $16(94)$ & $11(65)$ \\
\hline Leve & - & $3(18)$ \\
\hline Moderada & $1(6)$ & $1(6)$ \\
\hline Grave & - & $2(12)$ \\
\hline Total & $17(100)$ & $17(100)$ \\
\hline \multicolumn{3}{|c|}{ Depresión 3 meses poscirugía } \\
\hline \multicolumn{3}{|c|}{ Grado de depresión } \\
\hline Sin depresión & $14(82)$ & $9(53)$ \\
\hline Leve & $2(12)$ & $5(30)$ \\
\hline Moderada & $1(6)$ & $2(12)$ \\
\hline Grave & - & $1(5)$ \\
\hline Total & $17(100)$ & $17(100)$ \\
\hline$p$ & $0.03^{*+}$ & $<0.01^{* \dagger}$ \\
\hline
\end{tabular}

difíciles o invasivas (con implicación de cambios en el estilo de vida, ya sean temporales o permanentes), y además señalan que se debe informar a los pacientes sobre qué esperar del procedimiento desde el punto de vista físico y emocional. Por lo anterior, se procedió a realizar el análisis del impacto de los síntomas depresivos sobre diferentes aspectos y marcadores clínicos en presencia y ausencia de estos para la evolución de los pacientes.

Se evaluó la evolución de la EVA del dolor con medición en dos momentos, al mes y a los 3 meses de la intervención quirúrgica, y claramente se observó una tendencia a un menor grado de dolor en los pacientes que no tenían síntomas depresivos previos a la cirugía. Un ejemplo claro es el grupo sin síntomas depresivos de forma inicial, en el que hubo cuatro pacientes con dolor grave, y al mes y los 3 meses de la intervención ningún paciente presentaba dolor grave. Estadísticamente también se evidencia una diferencia significativa en la evolución de la EVA a favor del grupo que no tenía síntomas depresivos previos.

Es importante señalar que el dolor crónico de los pacientes tenía una media de $7 \pm 2$ meses previos a la intervención quirúrgica. El $20 \%$ de los pacientes con dolor crónico permanecen con dolor, sin cambios respecto a la percepción previa a la cirugía ${ }^{17}$. 
Al realizar el análisis de la evolución de nuestro grupo de estudio, en la escala mJOA se observa una diferencia significativa para ambos grupos; sin embargo, al analizarlo de forma detenida se ve que en el grupo sin síntomas depresivos solo el $29 \%$ tenían una mJOA grave al mes de la cirugía y el $11 \%$ a los 3 meses, contrastando con el grupo que presentaba síntomas depresivos previos a la cirugía, en el que el $47 \%$ tenía una mJOA grave al mes de la intervención y el $23 \%$ a los 3 meses, demostrando de nuevo que la depresión previa no tratada desempeña un papel muy importante en la evolución de la enfermedad y en la respuesta al tratamiento quirúrgico. En el grupo sin síntomas depresivos, al mes de la intervención el $94 \%$ continuó sin presentar datos de depresión y el $82 \%$ continuó así para el tercer mes. En el grupo con síntomas depresivos previos, se observó que al mes de la intervención el $64 \%$ no presentabna criterios diagnósticos de depresión y el $52 \%$ se mantuvo así hasta el tercer mes. Con lo anterior se evidencian unas relaciones positivas de la ausencia de depresión sobre la evolución de la enfermedad y de la intervención quirúrgica sobre la propia depresión, ambas de forma favorable.

Otro puntaje evaluado fue el NDI, con el cual se determinó nuevamente una diferencia significativa para ambos grupos, pero se encontró un valor de $p \leq 0.004$, lo que representa un intervalo de confianza superior al $99 \%$ para el grupo con síntomas depresivos. Se sugiere continuar con esta línea de investigación aumentando el análisis casuístico de esta variable.

Es importante comentar que en nuestro estudio hubo más hombres que mujeres con síntomas depresivos, y por lo general la depresión es más frecuente en las mujeres (3:1) que en los hombres; creemos que esto puede deberse al probable incremento de la esperanza de vida de los varones.

\section{Conclusiones}

Existe una relación favorable en los pacientes con MEC sometidos a tratamiento quirúrgico entre la ausencia de depresión previa a la cirugía y la evolución clínica. Se abre la puerta para promover un manejo multidisciplinario para que los pacientes con dicha patología y diagnosticados con depresión sean tratados previamente a la intervención quirúrgica, y además se sugiere una casuística mayor al respecto.
Se observó un impacto positivo de la cirugía por abordaje anterior sobre la evolución de la depresión en los pacientes con MEC.

Después del análisis realizado adoptamos la hipótesis verdadera, considerando que se cumplió el objetivo del estudio y se demostró que los pacientes con diagnóstico de MEC tratados quirúrgicamente mediante abordaje anterior que presentan depresión o ansiedad en el preoperatorio tendrán peores resultados clínicos y funcionales en el posquirúrgico que aquellos que no presentan ninguna alteración en el estado emocional, y la mayoría de los pacientes presentaron una velocidad de conducción nerviosa cervical que podría darnos la posibilidad de recuperación posquirúrgica.

\section{Agradecimientos}

Al servicio de psicología clínica y salud mental del Hospital de Traumatología, por sus aportaciones al artículo.

\section{Responsabilidades éticas}

Protección de personas y animales. Los autores declaran que para esta investigación no se han realizado experimentos en seres humanos ni en animales.

Confidencialidad de los datos. Los autores declaran que han seguido los protocolos de su centro de trabajo sobre la publicación de datos de pacientes.

Derecho a la privacidad y consentimiento informado. Los autores declaran que en este artículo no aparecen datos de pacientes.

\section{Financiamiento}

Los autores manifestamos que no hubo financiamiento externo ni patrocinios para la realización del estudio.

\section{Conflicto de intereses}

Los autores declaran no tener conflictos de intereses respecto al presente estudio.

\section{Bibliografía}

1. Kalsi-Ryan S, Karadimas SK, Felings MG. Cervical spondylotic myelopathy : the clinical phenomenon and an increasingly prevalent. Neurosci. 2014;19:409-21.

2. Karadimas SK, Erwin WM, Ely CC, Dettori JR, Fehlings MC. Pathophysiology and natural history of cervical spondylotic myelopathy. Spine (Phila Pa 1976). 2013;38(22 Suppl 1):S21-36. 
3. Nouri A, Tetreault L, Singh A, Karadimas SK, Fehlings MG. Degenerative cervical myelopathy: epidemiology, genetics, and pathogenesis. Spine (Phila Pa 1976). 2015;40:E675-93.

4. Boogaarts HD, Bartels RHMA. Prevalence of cervical spondylotic myelopathy. Euro Spine J. 2013;24(Suppl 2):139-41.

5. Northover JR, Wild JB, Braybrooke J, Blanco J. The epidemiology of cervical spondylotic myelopathy. Skeletal Radiol. 2012;41:1543-6.

6. Hukuda S, Mochizuki T, Ogata M, Shichikawa K, Shimomura Y. Operations for cervical spondylotic myelopathy. J Bone Joint Surg Br. 1985;67:609-15.

7. Nurjck S. The pathogenesis of the spinal cord disorder associated with cervical spondylosis (cervical spondylotic myelopathy, CSM) was originally attributed to compression of the cord by cartilaginous. Brain. 1972;95:87-100.

8. Andrade Ortega JA, Martínez ADD, Ruiz RA. Validación de una versión española del Índice de Discapacidad Cervical. Med Clin (Barc). 2008;130:85-9.

9. Rao RD, Gourab K, David KS. Operative treatment of cervical spondylotic myelopathy. J Bone Joint Surg. 2006;88:1619-40.

10. Riew KD, Buchowski JM, Sasso R, Zdeblick T, Metcalf NH, Anderson PA Cervical disc arthroplasty compared with arthrodesis for the treatment of myelopathy. J Bone Joint Surg. 2008;90:2354-64.
11. Bener A, Verjee M, Dafeeah EE, Falah O, Al-Juhaishi T, Schlogl J, et al. Psychological factors: anxiety, depression, and somatization symptoms in low back pain patients. J Pain Res. 2013;6:95-101.

12. Löbner M, Luppa M, Matschinger H, Konnopka A, Meisel HJ, Günther L, et al. The course of depression and anxiety in patients undergoing disc surgery: a longitudinal observational study. J Psychosom Res. 2012;72:185-94.

13. Pollock R, Lakkol S, Budithi C, Bhatia C, Krishna M. Effect of psychological status on outcome of posterior lumbar interbody fusion surgery. Asian Spine J. 2012;6:178-82.

14. Currie SR, Wang J. Chronic back pain and major depression in the general Canadian population. Pain. 2004;107:54-60.

15. González Caro C, Valencia Upegui H, Bersh Toros S. Intervenciones psicoterapéuticas en los pacientes con enfermedad médico-quirúrgica. Rev Colomb Psiquiatr. 2006;35:72-91.

16. Groves MS, Muskin PR. Psychological responses to illness. En: Levenson JL, editor. Textbook of psychosomatic medicine. Psychiatric care of the medically ill. $2^{\text {nd }}$ ed. Arlington, USA: The American Psychiatric Publishing; 2011. p. 45-67.

17. Aldrete JA, Ghaly RF. Actualización de las indicaciones quirúrgicas en el tratamiento del dolor crónico. Rev Soc Esp Dolor. 1998;5:35-51. 